\title{
Asynchronous Guessing Subject to Distortion
}

\author{
Shigeaki Kuzuoka \\ Wakayama University \\ Email: kuzuoka@ieee.org
}

\begin{abstract}
The problem of guessing subject to distortion is considered, and the performance of randomized guessing strategies is investigated. A one-shot achievability bound on the guessing moment (i.e., moment of the number of required queries) is given. Applying this result to i.i.d. sources, it is shown that randomized strategies can asymptotically attain the optimal guessing moment. Further, a randomized guessing scheme which is feasible even when the block size is extremely large is proposed, and a singleletter characterization of the guessing moment achievable by the proposed scheme is obtained.
\end{abstract}

\section{INTRODUCTION}

Consider the problem of guessing the realized value $x$ of a random variable $X$ using a sequence of queries of the form "Is $X=x$ ?". We are interested in how many queries are required until an affirmative answer is obtained; the number is called the guesswork. This guessing problem was introduced by Massey [1], where the expectation of guesswork was investigated. Subsequently Arikan [2] investigated the $\rho$-th moment $G_{\rho}$ of guesswork, which is called the guessing moment. Further, Arikan and Merhav [3] extended Arikan's result [2] to the lossy case, where the value $x$ of $X$ is not necessarily identified but it is required to find a value $\hat{x}$ satisfying $d(x, \hat{x}) \leq \Delta$ for given distortion measure $d$ and distortion level $\Delta$.

Recently asynchronous guessing problem was introduced by Salamatian et al. [4] as an information-theoretic model for brute-force botnet attacks. In the asynchronous setting, the guesser is restricted so that it does not know which queries were already asked. In [4], a modified variation $V_{\rho}$ of the guessing moment $G_{\rho}$ attainable by asynchronous guessing strategies is investigated. Their result implies that the optimal asynchronous guessing is given by randomized guessing, where the guesser choses a query according to a certain probability distribution, and that the penalty of lack of synchronization is asymptotically negligible.

The primary motivation of this work is to extend the study of [4] to the lossy case. Specifically, randomized guessing subject to distortion is studied. Our model provides a simplified mathematical model for brute-force attacks against bio-metrics authentication, where attacker's task is to find a query which is sufficiently similar to the bio-metric data stored in the system.

\section{A. Contributions}

Our first contribution is to give an achievability bound on $V_{\rho}$ in terms of a variation of the Rényi entropy (Theorem 11. In particular, when the order $\rho$ of the moment is an integer, we directly evaluate the guessing moment $G_{\rho}$ and give an achievability bound (Theorem 2 and its corollaries). Our achievability result reveals that there exists a deterministic quantizer $\pi$ which does not depend on the parameter $\rho$ and the optimal guessing strategy is given by the tilted distribution of the quantized $\hat{X}=\pi(X)$.

Next we apply our achievability bound to independent and identically distributed (i.i.d.) sources, and then, show that synchronization is not necessary to achieve the asymptotically optimal guessing moment. Furthermore, for asymptotic case, we propose i.i.d. asynchronous guessing strategies, which are simple and feasible even when the block size $n$ is extremely large. We investigate the asymptotic performance of i.i.d. asynchronous guessing strategies and give a single-letter characterization of the optimal guessing moment achievable by i.i.d. strategies (Theorem 3 and its corollary).

\section{B. Related Work}

The study of guessing was pioneered by Massey [1]. Arikan [2] demonstrated that the Rényi entropy [5] characterizes the guessing moment (up to some factor). Recently, tighter bounds on the guessing moment were given by Sason and Verdú [6].

The guessing problem has been studied in various contexts such as guessing allowing errors [7], guessing subject to distortion [3], [8], investigation of large deviation perspective of guessing [9], [10], guesswork in multi-user systems [11], and guesswork with distributed encoders [12] and so on.

Applications of guessing are around the information security, e.g., cracking passwords; See the introduction of [4] and Section II of [13] for review on guessing and security. To understand the impact of synchronization in botnet attacks, Salamatian et al. [4] proposed a simplified model for distributed brute-force attacks and introduced randomized guessing. Merhav and Cohen [13] studied randomized guessing under source uncertainty and proposed the universal randomized guessing strategy based on the LZ78 data compression algorithm [14]. The problem of randomized guessing under the individualsequence approach was also investigated by Merhav [15].

\section{Organization}

The rest of this paper is organized as follows. In Section II we introduce a variation of the Rényi entropy and its property. Section III describes our main results; one-shot results are given in Section $\amalg$ II-A and asymptotic results for i.i.d. sources are given in Section III-B All theorems are proved in Section IV Section $\mathrm{V}$ concludes the paper.

\section{PRELIMINARY}

Let $\mathcal{X}$ and $\hat{\mathcal{X}}$ be finite alphabets. Let $d: \mathcal{X} \times \hat{\mathcal{X}} \rightarrow[0, \infty)$ be a distortion measure and fix the distortion level $\Delta \geq 0$. 
For each $x \in \mathcal{X}$, let $\mathcal{A}_{\Delta}(x) \triangleq\{\hat{x} \in \hat{\mathcal{X}}: d(x, \hat{x}) \leq \Delta\}$. We assume that $\mathcal{A}_{\Delta}(x) \neq \emptyset$ for any $x \in \mathcal{X}$.

In the discussion of one-shot guessing, we will use the quantity $H_{\alpha}^{\Delta}(X)$, which was introduced in [8]. Let $\mathcal{W}_{\Delta}$ be the set of conditional distributions $P_{\hat{X} \mid X}$ such that $\operatorname{Pr}\{d(X, \hat{X}) \leq$ $\Delta\}=1$ (or equivalently $P_{\hat{X} \mid X}(\hat{x} \mid x)=0$ if $d(x, \hat{x})>\Delta$ ). Then $H_{\alpha}^{\Delta}(X)$ is defined as follows.

Definition 1: For $\alpha \in(0,1) \cup(1, \infty)$,

$$
H_{\alpha}^{\Delta}(X) \triangleq \inf _{P_{\hat{X} \mid X} \in \mathcal{W}_{\Delta}} H_{\alpha}(\hat{X})
$$

where $H_{\alpha}(\hat{X})$ is the Rényi entropy of $\left.\hat{X} \sim P_{\hat{X}} 1\right]$

$$
H_{\alpha}(\hat{X}) \triangleq \frac{1}{1-\alpha} \log \sum_{\hat{x} \in \hat{\mathcal{X}}}\left[P_{\hat{X}}(\hat{x})\right]^{\alpha} .
$$

As shown in Appendix A the infimum in (1) can be achieved by a deterministic quantizer.

Proposition 1: There exists a deterministic function $\pi: \mathcal{X} \rightarrow$ $\hat{\mathcal{X}}$ such that $\pi(x) \in \mathcal{A}_{\Delta}(x)$ for all $x \in \mathcal{X}$ and that $\hat{X}=\pi(X)$ satisfies, for all $\alpha \in(0,1)$,

$$
H_{\alpha}(\hat{X})=H_{\alpha}^{\Delta}(X) .
$$

\section{MAIN Results}

\section{A. One-Shot Bounds}

Let us consider a random variable $X \sim P_{X}$ on $\mathcal{X}$. We investigate the problem of guessing the realization value $x$ of $X$ subject to the distortion measure $d$.

An asynchronous guessing strategy is determined by a distribution $P_{\hat{X}}$ on $\hat{\mathcal{X}}$, which is independent of the realization $x$ of $X$ but may depend on $P_{X}$. The guesser continues to emit i.i.d. sequence of random variables $\hat{X}_{1}, \hat{X}_{2}, \ldots$ according to $P_{\hat{X}}$ as long as $d\left(x, \hat{X}_{i}\right)>D$. The number of guesses $G\left(x \mid P_{\hat{X}}\right)$ is given by the first index $k$ such that $d\left(x, \hat{X}_{k}\right) \leq \Delta$ or equivalently $\hat{X}_{k} \in \mathcal{A}_{\Delta}(x)$. It should be emphasized that, even when $X=x$ is fixed, the number $G\left(x \mid P_{\hat{X}}\right)$ of guesses is a random variable. It is easily seen that the distribution of $G\left(x \mid P_{\hat{X}}\right)$ is the geometric distribution with the parameter $P_{\hat{X}}\left(\mathcal{A}_{\Delta}(x)\right)$, i.e.,

$$
\operatorname{Pr}\left\{G\left(x \mid P_{\hat{X}}\right)=k\right\}=\left[1-P_{\hat{X}}\left(\mathcal{A}_{\Delta}(x)\right)\right]^{k-1} P_{\hat{X}}\left(\mathcal{A}_{\Delta}(x)\right) .
$$

Thus, for a given parameter $\rho>0$, the $\rho$-th moment of the number of guesses can be written as

$$
\mathbb{E}\left[G_{\rho}\left(X \mid P_{\hat{X}}\right)\right]=\sum_{x \in \mathcal{X}} P_{X}(x) G_{\rho}\left(x \mid P_{\hat{X}}\right)
$$

where 2

$$
G_{\rho}\left(x \mid P_{\hat{X}}\right) \triangleq \sum_{k=1}^{\infty} k^{\rho}\left[1-P_{\hat{X}}\left(\mathcal{A}_{\Delta}(x)\right)\right]^{k-1} P_{\hat{X}}\left(\mathcal{A}_{\Delta}(x)\right) .
$$

\footnotetext{
${ }^{1}$ Throughout the paper, log denotes the natural logarithm.

${ }^{2}$ Note that $G_{\rho}\left(x \mid P_{\hat{X}}\right)$ is not a random variable although $G\left(x \mid P_{\hat{X}}\right)$ is.
}

While our main interest is $\mathbb{E}\left[G_{\rho}\left(X \mid P_{\hat{X}}\right)\right]$, we first investigate the quantity

$$
V_{\rho}\left(x \mid P_{\hat{X}}\right) \triangleq \mathbb{E}\left[\left(\begin{array}{c}
G\left(x \mid P_{\hat{X}}\right)+\rho-1 \\
\rho
\end{array}\right)\right],
$$

where the expectation $\mathbb{E}$ is taken with respect to the random variable $G\left(x \mid P_{\hat{X}}\right)$ and $\left(\begin{array}{l}a \\ b\end{array}\right)$ is the generalized binomial coefficient defined in terms of the gamma function $\Gamma$, i.e.,

$$
\left(\begin{array}{l}
a \\
b
\end{array}\right)=\frac{\Gamma(a+1)}{\Gamma(b+1) \Gamma(a-b+1)} .
$$

The virtue of $V_{\rho}$ is that its value can be explicitly given as follows.

Proposition 2: For any guessing strategy $P_{\hat{X}}$ and $\rho>0$,

$$
V_{\rho}\left(x \mid P_{\hat{X}}\right)=\left(\frac{1}{P_{\hat{X}}\left(\mathcal{A}_{\Delta}(x)\right)}\right)^{\rho}, \quad \forall x \in \mathcal{X} .
$$

Corollary 1: For any $P_{\hat{X}}$ and $\rho>0$,

$$
\mathbb{E}\left[V_{\rho}\left(X \mid P_{\hat{X}}\right)\right]=\sum_{x \in \mathcal{X}} P_{X}(x)\left(\frac{1}{P_{\hat{X}}\left(\mathcal{A}_{\Delta}(x)\right)}\right)^{\rho} .
$$

The proposition is proved in Appendix B

Our one-shot achievability bound on $\mathbb{E}\left[V_{\rho}\left(X \mid P_{\hat{X}}\right)\right]$ is stated as follows.

Theorem 1: There exists a deterministic function $\pi: \mathcal{X} \rightarrow \hat{\mathcal{X}}$ such that, for all $\rho>0$, the tilted distribution

$$
P_{\hat{X}_{\rho}^{*}}(\hat{x}) \triangleq \frac{P_{\hat{X}}(\hat{x})^{\frac{1}{1+\rho}}}{\sum_{\hat{x}^{\prime}} P_{\hat{X}}\left(\hat{x}^{\prime}\right)^{\frac{1}{1+\rho}}}
$$

of the distribution $P_{\hat{X}}$ of $\hat{X}=\pi(X)$ satisfies

$$
\log \mathbb{E}\left[V_{\rho}\left(X \mid P_{\hat{X}_{\rho}^{*}}\right)\right] \leq \rho H_{\frac{1}{1+\rho}}^{\Delta}(X) .
$$

The theorem is proved in Section IV-A

Now we investigate our main interest, i.e., the $\rho$-th moment $\mathbb{E}\left[G_{\rho}\left(X \mid P_{\hat{X}}\right)\right]$ of the number of guesses. In particular, we consider the case where $\rho=1,2, \ldots$ is a positive integer 4 In this case, we can directly evaluate $G_{\rho}\left(x \mid P_{\hat{X}}\right)$ by using the moment generating function $M(t)=p_{x} \mathrm{e}^{t} /\left(1-\left(1-p_{x}\right) \mathrm{e}^{t}\right)$ of the geometric distribution with the parameter $p_{x} \triangleq P_{\hat{X}}\left(\mathcal{A}_{\Delta}(x)\right)$; e.g., the first four moments are

$$
\begin{aligned}
& G_{1}\left(x \mid P_{\hat{X}}\right)=1 / p_{x}, \\
& G_{2}\left(x \mid P_{\hat{X}}\right)=\left(2-p_{x}\right) / p_{x}^{2}, \\
& G_{3}\left(x \mid P_{\hat{X}}\right)=\left(p_{x}^{2}-6 p_{x}+6\right) / p_{x}^{3}, \\
& G_{4}\left(x \mid P_{\hat{X}}\right)=\left(-p_{x}^{3}+14 p_{x}^{2}-36 p_{x}+24\right) / p_{x}^{4} .
\end{aligned}
$$

Further, we have upper and lower bounds on $G_{\rho}\left(x \mid P_{\hat{X}}\right)$ as follows.

\footnotetext{
${ }^{3}$ The idea of considering the modification $V_{\rho}$ of the moment $G_{\rho}$ was introduced by Salamatian et al. [4].

${ }^{4}$ For non-integer $\rho>0$, we may numerically evaluate the $\rho$-th moment by using the technique recently developed in [16].
} 
Theorem 2: For any guessing strategy $P_{\hat{X}}$, any $x \in \mathcal{X}$, and any positive integer $\rho$,

$$
V_{\rho}\left(x \mid P_{\hat{X}}\right) \leq G_{\rho}\left(x \mid P_{\hat{X}}\right) \leq(\rho !) V_{\rho}\left(x \mid P_{\hat{X}}\right),
$$

or equivalently

$$
\left(\frac{1}{P_{\hat{X}}\left(\mathcal{A}_{\Delta}(x)\right)}\right)^{\rho} \leq G_{\rho}\left(x \mid P_{\hat{X}}\right) \leq(\rho !)\left(\frac{1}{P_{\hat{X}}\left(\mathcal{A}_{\Delta}(x)\right)}\right)^{\rho} .
$$

Corollary 2: For any $P_{\hat{X}}$ and positive integer $\rho$,

$$
\mathbb{E}\left[V_{\rho}\left(X \mid P_{\hat{X}}\right)\right] \leq \mathbb{E}\left[G_{\rho}\left(X \mid P_{\hat{X}}\right)\right] \leq(\rho !) \mathbb{E}\left[V_{\rho}\left(X \mid P_{\hat{X}}\right)\right]
$$

and

$$
\begin{aligned}
& \sum_{x \in \mathcal{X}} P_{X}(x)\left(\frac{1}{P_{\hat{X}}\left(\mathcal{A}_{\Delta}(x)\right)}\right)^{\rho} \\
& \leq \mathbb{E}\left[G_{\rho}\left(X \mid P_{\hat{X}}\right)\right] \\
& \leq(\rho !) \sum_{x \in \mathcal{X}} P_{X}(x)\left(\frac{1}{P_{\hat{X}}\left(\mathcal{A}_{\Delta}(x)\right)}\right)^{\rho} .
\end{aligned}
$$

The theorem is proved in Section IV-B.

From Theorems 1 and 2 we can obtain a one-shot achievability result in terms of $\mathbb{E}\left[G\left(X \mid P_{\hat{X}}\right)^{\rho}\right]$ as follows.

Corollary 3: There exists a deterministic function $\pi: \mathcal{X} \rightarrow$ $\hat{\mathcal{X}}$ such that, for any positive integer $\rho$, the tilted distribution $P_{\hat{X}_{\rho}^{*}}$ defined as (3) satisfies

$$
\log \mathbb{E}\left[G_{\rho}\left(X \mid P_{\hat{X}_{\rho}^{*}}\right)\right] \leq \rho H_{\frac{1}{1+\rho}}^{\Delta}(X)+\log (\rho !) .
$$

Let us compare our result with that of synchronous case. A synchronous guessing strategy is determined by a bijection $\mathcal{G}: \hat{\mathcal{X}} \rightarrow\{1,2, \ldots,|\hat{\mathcal{X}}|\}$, and the number of guesses when $X=x$ is given by

$$
G^{\text {sync }}(x \mid \mathcal{G}) \triangleq \min _{\hat{x} \in \mathcal{A}_{\Delta}(x)} \mathcal{G}(\hat{x}) .
$$

According to [8], the optimal $\rho$-th moment achievable by synchronous strategies satisfies

$$
\begin{aligned}
& \rho H_{\frac{1}{1+\rho}}^{\Delta}(X)-\rho \log \log (1+\min \{|\mathcal{X}|,|\hat{\mathcal{X}}|\}) \\
& \leq \log \min _{\mathcal{G}} \mathbb{E}\left[G^{\text {sync }}(X \mid \mathcal{G})^{\rho}\right] \\
& \leq \rho H_{\frac{1}{1+\rho}}^{\Delta}(X) .
\end{aligned}
$$

Comparing (4) with Corollary 3 , we can see that the penalty of lack of synchronization is upper bounded by

$$
\log (\rho !)+\rho \log \log (1+\min \{|\mathcal{X}|,|\hat{\mathcal{X}}|\}) .
$$

\section{B. Asymptotics for Stationary Memoryless Sources}

In this subsection, we apply our one-shot results to i.i.d. sources and investigate the asymptotic behavior of the $\rho$-th moment of the number of guesses 5

Let $\mathcal{X}^{n}$ (resp. $\hat{\mathcal{X}}^{n}$ ) is the $n$-fold Cartesian product of $\mathcal{X}$ (resp. $\hat{\mathcal{X}}$ ). The distortion between $\boldsymbol{x} \in \mathcal{X}^{n}$ and $\hat{\boldsymbol{x}} \in \hat{\mathcal{X}}^{n}$ per

\footnotetext{
${ }^{5}$ To simplify the argument, we assume that $\rho$ is a positive integer. However, it is not hard to show that our argument is valid for non-integer $\rho>0$; See Appendix $\mathbf{C}$
}

symbol is defined by $d_{n}(\boldsymbol{x}, \hat{\boldsymbol{x}})=(1 / n) \sum_{i=1}^{n} d\left(x_{i}, \hat{x}_{i}\right)$. We investigate the problem of guessing the realization value $x$ of $X^{n}=\left(X_{1}, X_{2}, \ldots, X_{n}\right)$ subject to the distortion measure $d_{n}$, where $X_{1}, \ldots, X_{n}$ are independently generated according to an identical distribution $P_{X}$ on $\mathcal{X}$.

As in the one-shot case, an asynchronous guessing strategy is determined by a distribution $P_{\hat{X}^{n}}$ on $\hat{\mathcal{X}}^{n}$. As a direct consequence of Corollary 3 , there exists a deterministic function $\pi_{n}: \mathcal{X}^{n} \rightarrow \hat{\mathcal{X}}^{n}$ such that the strategy $P_{\hat{X}_{\rho}^{* n}}$ induced by $\pi_{n}$ satisfies

$$
\frac{1}{n} \log \mathbb{E}\left[G_{\rho}\left(X^{n} \mid P_{\hat{X}_{\rho}^{* n}}\right)\right] \leq \frac{1}{n} \rho H_{\frac{1}{1+\rho}}^{\Delta}\left(X^{n}\right)+\zeta_{n}
$$

where $\zeta_{n} \triangleq(\rho !) / n \rightarrow 0$ as $n \rightarrow \infty$. This fact indicates that synchronization is not necessary to achieve the asymptotically optimal guessing moment.

However, the strategy $P_{\hat{X}_{\rho}^{* n}}$ may be not feasible when $n$ is large. In particular, it may not be easy to find and implement the function $\pi_{n}$. Hence, we restrict the class of guessing strategies.

Definition 2: An asynchronous guessing strategy $P_{\hat{X}^{n}}$ is said to be an i.i.d. asynchronous guessing strategy if there exists a distribution $Q_{\hat{X}}$ on $\hat{\mathcal{X}}$ satisfying

$$
P_{\hat{X}^{n}}(\hat{\boldsymbol{x}})=Q_{\hat{X}}^{n}(\boldsymbol{x}) \triangleq \prod_{i=1}^{n} Q_{\hat{X}}\left(\hat{x}_{i}\right), \quad \forall \hat{\boldsymbol{x}} \in \hat{\mathcal{X}}^{n} .
$$

In the following, we investigate the optimal guessing moment asymptotically achievable by i.i.d. asynchronous guessing strategies. To state our result, we introduce some notation. We use the following standard information-theoretic quantities [17]. For a distribution $P$ and a conditional distribution $V$, let $H(P)$ be the entropy of $P, H(V \mid P)=\sum_{x} P(x) H(V(\cdot \mid x))$ be the conditional entropy, and $I(P, V)=H(P V)-H(V \mid P)$ be the mutual information, where $P V$ is the distribution such that $P V(\hat{x})=\sum_{x} P(x) V(\hat{x} \mid x)$. For two distributions $P$ and $Q$, let $D(P \| Q)$ be the divergence between $P$ and $Q$. Let $\overline{\mathcal{W}}_{\Delta}\left(Q_{X}\right)$ be the set of conditional distributions satisfying $\sum_{x, \hat{x}} Q_{X}(x) V(\hat{x} \mid x) d(x, \hat{x}) \leq \Delta$.

Definition 3: For distributions $Q_{X}$ on $\mathcal{X}$ and $Q_{\hat{X}}$ on $\hat{\mathcal{X}}$,

$$
R\left(Q_{X}, Q_{\hat{X}} \mid \Delta\right) \triangleq \min _{V \in \overline{\mathcal{W}}_{\Delta}\left(Q_{X}\right)}\left[I\left(Q_{X}, V\right)+D\left(Q_{X} V \| Q_{\hat{X}}\right)\right]
$$

where $Q_{X} V$ is the distribution such that $Q_{X} V(\hat{x})=$ $\sum_{x} Q_{X}(x) V(\hat{x} \mid x)$.

The next theorem, which is proved in Section IV-C, is our main result of this subsection.

Theorem 3: For any i.i.d. asynchronous guessing strategy $Q_{\hat{X}}$ and positive integer $\rho$,

$$
\begin{aligned}
& \lim _{n \rightarrow \infty} \frac{1}{n} \log \mathbb{E}\left[G_{\rho}\left(X^{n} \mid Q_{\hat{X}}^{n}\right)\right] \\
& =\max _{Q_{X}}\left[\rho R\left(Q_{X}, Q_{\hat{X}} \mid \Delta\right)-D\left(Q_{X} \| P_{X}\right)\right]
\end{aligned}
$$

where the maximum is taken over all distributions $Q_{X}$ on $\mathcal{X}$. 
As a consequence of the theorem, we obtain the following result on the optimal $\rho$-th moment achievable by i.i.d. asynchronous guessing strategies.

Corollary 4: The exponent of the optimal $\rho$-th moment achievable by i.i.d. asynchronous guessing strategies is

$$
E_{\rho}^{\text {i.i.d }}\left(P_{X} \mid \Delta\right) \triangleq \min _{Q_{\hat{X}}} \max _{Q_{X}}\left[\rho R\left(Q_{X}, Q_{\hat{X}}\right)-D\left(Q_{X} \| P_{X}\right)\right]
$$

where min (resp. max) is taken over all distributions $Q_{\hat{X}}$ on $\hat{\mathcal{X}}$ (resp. $Q_{X}$ on $\mathcal{X}$ ).

Remark 1: The corollary guarantees that we can find the optimal i.i.d. strategy by solving the minimization in the definition of $E_{\rho}^{\text {i.i.d }}\left(P_{X} \mid \Delta\right)$, which does not depend on $n$. So, our strategy is feasible even when $n$ is extremely large.

Remark 2: It should be emphasized that Theorem 3 holds for any strategy $Q_{\hat{X}}$. In other words, $Q_{\hat{X}}$ is not necessarily depend on $P_{X}$. Hence, it can be easily applied to guessing under source uncertainty. Assume that the guesser does not know the source distribution $P_{X}$ but it knows the fact that $P_{X} \in \mathcal{P}$ for a subset $\mathcal{P}$ of distributions. Theorem 3 shows that, under this setting, the exponent of the optimal $\rho$-th guessing moment asymptotically achievable by i.i.d. strategies is

$$
\min _{Q_{\hat{X}}} \max _{P_{X} \in \mathcal{P}} \max _{Q_{X}}\left[\rho R\left(Q_{X}, Q_{\hat{X}}\right)-D\left(Q_{X} \| P_{X}\right)\right] .
$$

Lastly, we investigate the penalty of restricting strategies to be i.i.d.

Let us define

$$
E_{\rho}\left(P_{X} \mid \Delta\right) \triangleq \max _{Q_{X}}\left\{\rho R\left(Q_{X} \mid \Delta\right)-D\left(Q_{X} \| P_{X}\right)\right\}
$$

where the maximum is taken over all distributions $Q_{X}$ on $\mathcal{X}$ and $R\left(Q_{X} \mid \Delta\right)$ is the rate-distortion function; i.e.,

$$
R\left(Q_{X} \mid \Delta\right) \triangleq \min _{V \in \overline{\mathcal{W}}_{\Delta}\left(Q_{X}\right)} I\left(Q_{X}, V\right)
$$

It is known that $E_{\rho}\left(P_{X} \mid \Delta\right)$ is the exponent of the optimal $\rho$ th guessing moment asymptotically achievable by synchronous strategies [3]; i.e.,

$$
E_{\rho}\left(P_{X} \mid \Delta\right)=\lim _{n \rightarrow \infty} \frac{1}{n} \log \min _{\mathcal{G}_{n} \text { on } \hat{\mathcal{X}}^{n}} \mathbb{E}\left[G^{\text {sync }}\left(X^{n} \mid \mathcal{G}_{n}\right)^{\rho}\right] .
$$

Further, results of [8] implies that

$$
E_{\rho}\left(P_{X} \mid \Delta\right)=\lim _{n \rightarrow \infty} \frac{\rho}{n} H_{\frac{1}{1+\rho}}^{\Delta}\left(X^{n}\right) .
$$

On the other hand, since

$$
\min _{Q_{\hat{X}}} R\left(Q_{X}, Q_{\hat{X}} \mid \Delta\right)=R\left(Q_{X} \mid \Delta\right),
$$

we have

$$
\begin{aligned}
E_{\rho}^{\text {i.i.d }}\left(P_{X} \mid \Delta\right) & =\min _{Q_{\hat{X}}} \max _{Q_{X}}\left[\rho R\left(Q_{X}, Q_{\hat{X}} \mid \Delta\right)-D\left(Q_{X} \| P_{X}\right)\right] \\
& \geq \max _{Q_{X}} \min _{Q_{\hat{X}}}\left[\rho R\left(Q_{X}, Q_{\hat{X}} \mid \Delta\right)-D\left(Q_{X} \| P_{X}\right)\right] \\
& =E_{\rho}\left(P_{X} \mid \Delta\right) .
\end{aligned}
$$

From this, we can see the suboptimality of i.i.d. strategies and evaluate the penalty as

$$
\begin{aligned}
& \min _{Q_{\hat{X}}} \max _{Q_{X}}\left[\rho R\left(Q_{X}, Q_{\hat{X}} \mid \Delta\right)-D\left(Q_{X} \| P_{X}\right)\right] \\
& -\max _{Q_{X}} \min _{Q_{\hat{X}}}\left[\rho R\left(Q_{X}, Q_{\hat{X}} \mid \Delta\right)-D\left(Q_{X} \| P_{X}\right)\right] .
\end{aligned}
$$

\section{PROOFS}

\section{A. Proof of Theorem 1}

Let $\pi$ be the function given in Proposition 1 and let $\hat{X}=\pi(X)$. Since $\pi(x) \in \mathcal{A}_{\Delta}(x)$, we have $P_{\hat{X}_{\rho}^{*}}\left(\mathcal{A}_{\Delta}(x)\right) \geq$ $P_{\hat{X}_{\rho}^{*}}(\pi(x))$. Hence, letting $\pi^{-1}(\hat{x}) \triangleq\{x \in \mathcal{X}: \pi(x)=\hat{x}\}$, we have

$$
\begin{aligned}
\mathbb{E}\left[V_{\rho}\left(X \mid P_{\hat{X}_{\rho}^{*}}\right)\right] & =\sum_{x \in \mathcal{X}} P_{X}(x)\left(\frac{1}{P_{\hat{X}_{\rho}^{*}}\left(\mathcal{A}_{\Delta}(x)\right)}\right)^{\rho} \\
& \leq \sum_{x \in \mathcal{X}} P_{X}(x)\left(\frac{1}{P_{\hat{X}_{\rho}^{*}}(\pi(x))}\right)^{\rho} \\
& =\sum_{\hat{x} \in \hat{\mathcal{X}}} \sum_{x \in \pi^{-1}(\hat{x})} P_{X}(x)\left(\frac{1}{P_{\hat{X}_{\rho}^{*}}(\hat{x})}\right)^{\rho} \\
& =\sum_{\hat{x} \in \hat{\mathcal{X}}} P_{\hat{X}}(\hat{x})\left(\frac{1}{P_{\hat{X}_{\rho}^{*}}(\hat{x})}\right)^{\rho} \\
& =\exp \left[\rho H_{\frac{1}{1+\rho}}(\hat{X})\right] \\
& =\exp \left[\rho H_{\frac{1}{1+\rho}}^{\Delta}(X)\right]
\end{aligned}
$$

where the last equality follows from (2).

\section{B. Proof of Theorem 2}

Since $\rho>1$, using Jensen's inequality, we have

$$
\begin{aligned}
G_{\rho}\left(x \mid P_{\hat{X}}\right) & =\sum_{k=1}^{\infty} \operatorname{Pr}\left\{G\left(x \mid P_{\hat{X}}\right)=k\right\} k^{\rho} \\
& \geq\left\{\sum_{k=1}^{\infty} \operatorname{Pr}\left\{G\left(x \mid P_{\hat{X}}\right)=k\right\} k\right\}^{\rho} \\
& =\left\{1 / P_{\hat{X}}\left(\mathcal{A}_{\Delta}(x)\right)\right\}^{\rho}
\end{aligned}
$$

where the last equality follows from the fact that the distribution of $G\left(x \mid P_{\hat{X}}\right)$ is the geometric distribution with the parameter $P_{\hat{X}}\left(\mathcal{A}_{\Delta}(x)\right)$.

On the other hand, since $G\left(x \mid P_{\hat{X}}\right)$ is an integer-valued random variable and $\rho$ is an integer,

$$
\begin{aligned}
\frac{G\left(x \mid P_{\hat{X}}\right)^{\rho}}{\rho !} \leq & \frac{1}{\rho !} G\left(x \mid P_{\hat{X}}\right) \times\left[G\left(x \mid P_{\hat{X}}\right)+1\right] \\
& \times\left[G\left(x \mid P_{\hat{X}}\right)+2\right] \times \cdots \times\left[G\left(x \mid P_{\hat{X}}\right)+\rho-1\right] \\
= & \left(\begin{array}{c}
G\left(x \mid P_{\hat{X}}\right)+\rho-1 \\
\rho
\end{array}\right) .
\end{aligned}
$$

Taking the expectation with respect to $G\left(x \mid P_{\hat{X}}\right)$ and multiply both sides by $\rho$ !, we have

$$
G_{\rho}\left(x \mid P_{\hat{X}}\right) \leq(\rho !) V_{\rho}\left(x \mid P_{\hat{X}}\right) .
$$

Combining (5) and (6) with Proposition 2, we have the theorem. 


\section{Proof of Theorem 3}

Before giving the proof, we introduce some notation.

For two positive sequences $a_{n}$ and $b_{n}$, we will write $a_{n} \doteq b_{n}$ if $\lim _{n \rightarrow \infty}(1 / n) \log \left(a_{n} / b_{n}\right)=0$. Similarly, when $a_{n}$ and $b_{n}$ depend on a sequence $\boldsymbol{x}$, the notation $a_{n}(\boldsymbol{x}) \doteq b_{n}(\boldsymbol{x})$ means that

$$
\lim _{n \rightarrow \infty} \max _{\boldsymbol{x} \in \mathcal{X}^{n}}\left|\frac{1}{n} \log \frac{a_{n}(\boldsymbol{x})}{b_{n}(\boldsymbol{x})}\right|=0 .
$$

In our proof, we use the method of types [17]. For $\boldsymbol{x} \in \mathcal{X}^{n}$, the type $P_{\boldsymbol{x}}$ is the empirical distribution of $\boldsymbol{x}=\left(x_{1}, \ldots, x_{n}\right)$; i.e., $P_{\boldsymbol{x}}(a)=(1 / n)\left|\left\{1 \leq i \leq n: x_{i}=a\right\}\right|$ for all $a \in \mathcal{X}$. Let $\mathcal{P}_{n}$ be the possible types of length $n$ sequences. For $Q \in \mathcal{P}_{n}, \mathcal{T}_{Q}$ is the set of sequences $\boldsymbol{x}$ such that $P_{\boldsymbol{x}}=Q$. For a conditional distribution $V: \mathcal{X} \rightarrow \hat{\mathcal{X}}$ and $\boldsymbol{x} \in \mathcal{X}^{n}$, $\mathcal{T}_{V}(\boldsymbol{x})$ denotes the set of sequences $\hat{\boldsymbol{x}}=\left(\hat{x}_{1}, \ldots, \hat{x}_{n}\right) \in \hat{\mathcal{X}}^{n}$ satisfying $V(b \mid a)=\left|\left\{j:\left(x_{j}, \hat{x}_{j}\right)=(a, b)\right\}\right| /\left|\left\{i: x_{i}=a\right\}\right|$ for all $(a, b) \in \mathcal{X} \times \hat{\mathcal{X}}$.

Proof of Theorem 3 . From Corollary 2] we have

$$
\mathbb{E}\left[G_{\rho}\left(X^{n} \mid Q_{\hat{X}}^{n}\right)\right] \doteq \sum_{\boldsymbol{x} \in \mathcal{X}^{n}} P_{X^{n}}(\boldsymbol{x})\left[Q_{\hat{X}}^{n}\left(\mathcal{A}_{\Delta}(\boldsymbol{x})\right)\right]^{-\rho}
$$

where $\mathcal{A}_{\Delta}(\boldsymbol{x}) \triangleq\left\{\hat{\boldsymbol{x}} \in \hat{\mathcal{X}}^{n}: d_{n}(\boldsymbol{x}, \hat{\boldsymbol{x}}) \leq \Delta\right\}$. So, we evaluate the exponent of the right-hand side of (7).

For any $\hat{\boldsymbol{x}}$ and $V$, we have

$$
\begin{aligned}
Q_{\hat{X}}^{n}(\hat{\boldsymbol{x}}) & =\exp \left\{-n\left[H\left(P_{\hat{\boldsymbol{x}}}\right)+D\left(P_{\hat{\boldsymbol{x}}} \| Q_{\hat{X}}\right)\right]\right\}, \\
\left|\mathcal{T}_{V}(\boldsymbol{x})\right| & \doteq \exp \left\{n H\left(V \mid P_{\boldsymbol{x}}\right)\right\}
\end{aligned}
$$

and thus,

$$
Q_{\hat{X}}^{n}\left(\mathcal{T}_{V}(\boldsymbol{x})\right) \doteq \exp \left\{-n\left[I\left(P_{\boldsymbol{x}}, V\right)+D\left(P_{\boldsymbol{x}} V \| Q_{\hat{X}}\right)\right]\right\}
$$

where $P_{\boldsymbol{x}} V$ is the distribution on $\hat{\mathcal{X}}$ such that $P_{\boldsymbol{x}} V(\hat{x})=$ $\sum_{x} P_{\boldsymbol{x}}(x) V(\hat{x} \mid x)$.

Further, $\mathcal{A}_{\Delta}(\boldsymbol{x})$ can be written as

$$
\mathcal{A}_{\Delta}(\boldsymbol{x})=\underset{\sum_{x, \hat{x}} P_{\boldsymbol{x}}(x) V\left(\dot{\hat{x}}^{\prime} \mid x\right) d(x, \hat{x}) \leq \Delta}{\bigcup_{V}(\boldsymbol{x}) .}
$$

Hence, we have

$$
\begin{aligned}
Q_{\hat{X}}^{n}\left(\mathcal{A}_{\Delta}(\boldsymbol{x})\right) & \doteq \exp \left\{-n \min _{V}\left[I\left(P_{\boldsymbol{x}}, V\right)+D\left(P_{\boldsymbol{x}} V \| Q_{\hat{X}}\right)\right]\right\} \\
& =\exp \left\{-n R\left(P_{\boldsymbol{x}}, Q_{\hat{X}} \mid \Delta\right)\right\} .
\end{aligned}
$$

On the other hand, we have

$$
P_{X}\left(\mathcal{T}_{Q_{X}}\right) \doteq \exp \left\{-n D\left(Q_{X} \| P_{X}\right)\right\}
$$

for all $Q_{X} \in \mathcal{P}_{n}$. Combining this with (8), we have

$$
\begin{aligned}
& \sum_{\boldsymbol{x} \in \mathcal{X}^{n}} P_{X^{n}}(\boldsymbol{x})\left[Q_{\hat{X}}^{n}\left(\mathcal{A}_{\Delta}(\boldsymbol{x})\right)\right]^{-\rho} \\
& =\sum_{Q_{X} \in \mathcal{P}_{n}} \sum_{\boldsymbol{x} \in \mathcal{T}_{Q_{X}}} P_{X^{n}}(\boldsymbol{x})\left[Q_{\hat{X}}^{n}\left(\mathcal{A}_{\Delta}(\boldsymbol{x})\right)\right]^{-\rho} \\
\doteq & \sum_{Q_{X} \in \mathcal{P}_{n}} \exp \left\{n\left[\rho R\left(Q_{X}, Q_{\hat{X}} \mid \Delta\right)-D\left(Q_{X} \| P_{X}\right)\right]\right\} \\
\doteq & \exp \left\{n \max _{Q_{X}}\left[\rho R\left(Q_{X}, Q_{\hat{X}} \mid \Delta\right)-D\left(Q_{X} \| P_{X}\right)\right]\right\} .
\end{aligned}
$$

\section{CONCLUding REMARKS}

In this paper, randomized strategies for guessing subject to distortion was studied. A one-shot achievability bound on the guessing moment was given. Further, feasible i.i.d. asynchronous guessing scheme was proposed, and its asymptotic performance was investigated.

Lastly, we give some comments regarding generalizations of our results.

- It is not hard to extend the result to the case where sideinformation is available at the guesser.

- Our result shows that the behavior of

$$
-\frac{1}{n} \log Q_{\hat{X}}^{n}\left(\mathcal{A}_{\Delta}(\boldsymbol{x})\right) \simeq R\left(P_{\boldsymbol{x}}, Q_{\hat{X}} \mid \Delta\right)
$$

determines the guessing moment (See (8) and (7) in the proof of Theorem 3). Since the behavior of $(-1 / n) \log Q_{\hat{X}}^{n}\left(\mathcal{A}_{\Delta}(\boldsymbol{x})\right)$ for sources with memory is well studied in the context of the rate-distortion theory (see [18] and references there in), we can apply those results. For example, our argument can also be applied to stationary ergodic sources by using Theorem 3 of [19].

\section{APPENDIX A}

\section{ProOF OF PROPOSITION 1}

First we introduce the concept of majorization and Schur concavity, which play important role in the proof.

Let $\mathbb{R}_{+}^{m}$ be the set of vectors with $m$ nonnegative components. Given $\mathbf{p}=\left(p_{1}, p_{2}, \ldots, p_{m}\right) \in \mathbb{R}_{+}^{m}$, denote by $p_{[1]} \geq p_{[2]} \geq \cdots \geq p_{[m]}$ the permutation of the components of $\mathbf{p}$ in the nonincreasing order.

Definition 4: We say that $\mathbf{q} \in \mathbb{R}_{+}^{m}$ majorizes $\mathbf{p} \in \mathbb{R}_{+}^{m}$ (and write $\mathbf{p} \prec \mathbf{q}$ ) if

$$
\sum_{i=1}^{j} p_{[i]} \leq \sum_{i=1}^{j} q_{[i]}, \quad \forall j=1,2, \ldots, m-1
$$

and

$$
\sum_{i=1}^{m} p_{[i]} \leq \sum_{i=1}^{m} q_{[i]} .
$$

Definition 5: A real valued function $h$ on $\mathbb{R}_{+}^{m}$ is said to be Schur concave if $h(\mathbf{p}) \geq h(\mathbf{q})$ for any $\mathbf{p}, \mathbf{q} \in \mathbb{R}_{+}^{m}$ satisfying $\mathbf{p} \prec \mathbf{q}$.

It is well known that $\phi(\mathbf{p})=\sum_{i=1}^{m}\left(h_{i}\right)^{\alpha}$ for $\alpha \in(0,1)$ is Schur concave; See, e.g. [20]. Thus, we can easily see that the Rényi entropy of order $\alpha \in(0,1)$ is also Schur concave. Hence, to prove Proposition 11, it is sufficient to prove the following lemma. Although the same argument is given in the last page of [21], we give a proof for the completeness.

Lemma 1: There exists a deterministic function $\pi: \mathcal{X} \rightarrow \hat{\mathcal{X}}$ such that (i) $d(x, \pi(x)) \leq \Delta(x)$ for all $x \in \mathcal{X}$ and (ii) the distribution $P_{\hat{X}}$ of $\hat{X}=\pi(X)$ majorizes any $P_{\tilde{X}}$ induced by $P_{\tilde{X} \mid X} \in \mathcal{W}_{\Delta}$.

Proof: Let $m \triangleq|\mathcal{X}|$ in this proof. For each $\hat{x} \in \mathcal{X}$, let

$$
\mathcal{B}_{\Delta}(\hat{x}) \triangleq\{x \in \mathcal{X}: d(x, \hat{x}) \leq \Delta\} .
$$


We define the order $\hat{x}_{1}, \hat{x}_{2}, \ldots, \hat{x}_{m}$ of symbols in $\hat{\mathcal{X}}$ as follows. Let $\hat{x}_{1}$ be a symbol satisfying

$$
\operatorname{Pr}\left\{X \in \mathcal{B}_{\Delta}\left(\hat{x}_{1}\right)\right\}=\max _{\hat{x} \in \hat{\mathcal{X}}} \operatorname{Pr}\left\{X \in \mathcal{B}_{\Delta}(\hat{x})\right\} .
$$

Then, for $i=2,3, \ldots, m$, let $\hat{x}_{i} \in \hat{\mathcal{X}} \backslash\left\{\hat{x}_{1}, \ldots, \hat{x}_{i-1}\right\}$ be a symbol such that

$$
\begin{aligned}
& \operatorname{Pr}\left\{X \in \mathcal{B}_{\Delta}\left(\hat{x}_{i}\right) \backslash \bigcup_{j=1}^{i-1} \mathcal{B}_{\Delta}\left(\hat{x}_{j}\right)\right\} \\
& =\max _{\hat{x} \in \hat{\mathcal{X}}} \operatorname{Pr}\left\{X \in \mathcal{B}_{\Delta}(\hat{x}) \backslash \bigcup_{j=1}^{i-1} \mathcal{B}_{\Delta}\left(\hat{x}_{j}\right)\right\} .
\end{aligned}
$$

Let $\mathcal{X}_{i} \triangleq \mathcal{B}_{\Delta}\left(\hat{x}_{i}\right) \backslash \bigcup_{j=1}^{i-1} \mathcal{B}_{\Delta}\left(\hat{x}_{j}\right)(i=1, \ldots, m)$. Then $\mathcal{X}_{1}, \ldots, \mathcal{X}_{m}$ give a partition of $\mathcal{X}$, and thus, we can define $\pi: \mathcal{X} \rightarrow \hat{\mathcal{X}}$ so that $\pi(x)=\hat{x}_{i}$ if $x \in \mathcal{X}_{i}$. It is apparent that $d(x, \pi(x)) \leq \Delta$ for all $x \in \mathcal{X}$. Further, from the construction, the distribution $P_{\hat{X}}$ of $\hat{X}=\pi(X)$ satisfies

$$
P_{\hat{X}}\left(\hat{x}_{1}\right) \geq P_{\hat{X}}\left(\hat{x}_{2}\right) \geq \cdots \geq P_{\hat{X}}\left(\hat{x}_{m}\right) .
$$

We will prove that $\pi$ satisfies (ii) by contradiction. Assume that there exists $P_{\tilde{X} \mid X} \in \mathcal{W}_{\Delta}$ such that $P_{\tilde{X}}$ induced by $P_{\tilde{X} \mid X}$ is not majorized by $P_{\hat{X}}$. Let us define another order $\tilde{x}_{1}, \tilde{x}_{2}, \ldots, \tilde{x}_{m}$ in $\hat{\mathcal{X}}$ so that

$$
P_{\tilde{X}}\left(\tilde{x}_{1}\right) \geq P_{\tilde{X}}\left(\tilde{x}_{2}\right) \geq \cdots \geq P_{\tilde{X}}\left(\tilde{x}_{m}\right) .
$$

Since $P_{\tilde{X}} \nprec P_{\hat{X}}$, there exists $k$ such that

$$
\sum_{i=1}^{k} P_{\tilde{X}}\left(\tilde{x}_{i}\right)>\sum_{i=1}^{k} P_{\hat{X}}\left(\hat{x}_{i}\right) .
$$

Hence, we have

$$
\begin{aligned}
& \operatorname{Pr}\{d(X, \tilde{X})>\Delta\} \\
& \geq \sum_{x \in \mathcal{X}} \sum_{i=1}^{k} P_{X}(x) P_{\tilde{X} \mid X}\left(\tilde{x}_{i} \mid x\right) \mathbf{1}\left[d\left(x, \tilde{x}_{i}\right)>\Delta\right] \\
& =\sum_{i=1}^{k} P_{\tilde{X}}\left(\tilde{x}_{i}\right) \\
& -\sum_{x \in \mathcal{X}} P_{X}(x) \sum_{i=1}^{k} P_{\tilde{X} \mid X}\left(\tilde{x}_{i} \mid x\right) \mathbf{1}\left[x \in \mathcal{B}_{\Delta}\left(\tilde{x}_{i}\right)\right] \\
& \stackrel{\text { (a) }}{\geq} \sum_{i=1}^{k} P_{\tilde{X}}\left(\tilde{x}_{i}\right)-\operatorname{Pr}\left\{X \in \bigcup_{i=1}^{k} \mathcal{B}_{\Delta}\left(\tilde{x}_{i}\right)\right\} \\
& \stackrel{\text { (b) }}{\geq} \sum_{i=1}^{k} P_{\tilde{X}}\left(\tilde{x}_{i}\right)-\operatorname{Pr}\left\{X \in \bigcup_{i=1}^{k} \mathcal{B}_{\Delta}\left(\hat{x}_{i}\right)\right\} \\
& =\sum_{i=1}^{k} P_{\tilde{X}}\left(\tilde{x}_{i}\right)-\sum_{i=1}^{k} \operatorname{Pr}\left\{X \in \mathcal{X}_{i}\right\} \\
& =\sum_{i=1}^{k} P_{\tilde{X}}\left(\tilde{x}_{i}\right)-\sum_{i=1}^{k} P_{\hat{X}}\left(\hat{x}_{i}\right) \\
& >0 \text {, }
\end{aligned}
$$

where 1 denotes the indicator function, (a) follows from $\sum_{i=1}^{k} P_{\tilde{X} \mid X}\left(\tilde{x}_{i} \mid x\right) \mathbf{1}\left[x \in \mathcal{B}_{\Delta}\left(\tilde{x}_{i}\right)\right] \leq \mathbf{1}\left[x \in \bigcup_{i=1}^{k} \mathcal{B}_{\Delta}\left(\tilde{x}_{i}\right)\right]$ for all $x \in \mathcal{X}$, and (b) follows from the definition of the order $\hat{x}_{1}, \hat{x}_{2}, \ldots, \hat{x}_{m}$. This contradicts the fact $P_{\tilde{X} \mid X} \in \mathcal{W}_{\Delta}$.

\section{APPENDIX B}

Proof of Proposition 2

The proposition can be proved in the same manner as [4 Lemma 2]. We give a proof for the completeness.

Proof of Proposition 2. Letting $p_{x} \triangleq P_{\hat{X}}\left(\mathcal{A}_{\Delta}(x)\right)$, we have

$$
\begin{aligned}
V_{\rho}\left(x \mid P_{\hat{X}}\right) & =\sum_{m=1}^{\infty}\left(\begin{array}{c}
m+\rho-1 \\
\rho
\end{array}\right) \operatorname{Pr}\left\{G\left(x \mid P_{\hat{X}}\right)=m\right\} \\
& =p_{x} \sum_{m=1}^{\infty}\left(\begin{array}{c}
m+\rho-1 \\
\rho
\end{array}\right)\left(1-p_{x}\right)^{m-1} \\
& \stackrel{(a)}{=} p_{x} \sum_{m=1}^{\infty}\left(\begin{array}{c}
-\rho-1 \\
m-1
\end{array}\right)\left[-\left(1-p_{x}\right)\right]^{m-1} \\
& =p_{x} \sum_{k=0}^{\infty}\left(\begin{array}{c}
-\rho-1 \\
k
\end{array}\right)\left[-\left(1-p_{x}\right)\right]^{k} \\
& \stackrel{(\mathrm{b})}{=} p_{x}\left[1-\left(1-p_{x}\right)\right]^{-\rho-1} \\
& =\left(p_{x}\right)^{-\rho}
\end{aligned}
$$

where (a) follows from the relationship

$$
\left(\begin{array}{c}
m+\rho-1 \\
\rho
\end{array}\right)=(-1)^{m-1}\left(\begin{array}{c}
-\rho-1 \\
m-1
\end{array}\right),
$$

which is proved in the proof of Lemma 2 in [4], and (b) follows from the binominal formula.

\section{APPENDIX C}

\section{ASYMPTOTICS FOR NON-INTEGER $\rho$}

We show that Theorem 3 also holds for non-integer $\rho>0$. From (8), for all $\boldsymbol{x} \in \mathcal{X}^{n}$ and $Q_{\hat{X}}$,

$$
q_{\boldsymbol{x}} \triangleq Q_{\hat{X}}^{n}\left(\mathcal{A}_{\Delta}(\boldsymbol{x})\right) \doteq \exp \left\{-n R\left(P_{\boldsymbol{x}}, Q_{\hat{X}} \mid \Delta\right)\right\} .
$$

Assume that $R\left(P_{\boldsymbol{x}}, Q_{\hat{X}} \mid \Delta\right)>0$. Then, since $q_{\boldsymbol{x}}<1 / 2$ for large $n,(20)$ of [15] gives

$$
\begin{aligned}
G_{\rho}\left(\boldsymbol{x} \mid Q_{\hat{X}}^{n}\right) & =\sum_{k=1}^{\infty} k^{\rho}\left(1-q_{\boldsymbol{x}}\right)^{k-1} q_{\boldsymbol{x}} \\
& \geq\left(\frac{1-q_{\boldsymbol{x}}}{q_{\boldsymbol{x}}}\right)^{\rho} \exp \left\{-\frac{1}{1-q_{\boldsymbol{x}}}\right\} \\
& \geq \frac{2^{-\rho}}{\mathrm{e}^{2}} q_{\boldsymbol{x}}^{-\rho} .
\end{aligned}
$$

Thus, we have

$$
\liminf _{n \rightarrow \infty} \frac{1}{n} \log \left|\frac{G_{\rho}\left(\boldsymbol{x} \mid Q_{\hat{X}}^{n}\right)}{\exp \left\{\rho n R\left(P_{\boldsymbol{x}}, Q_{\hat{X}} \mid \Delta\right)\right\}}\right| \geq 0 .
$$

On the other hand, by using Lemma 1 of [13] with $a=$ $R\left(P_{\boldsymbol{x}}, Q_{\hat{X}} \mid \Delta\right)$, we can show that

$$
\limsup _{n \rightarrow \infty} \frac{1}{n} \log \left|\frac{G_{\rho}\left(\boldsymbol{x} \mid Q_{\hat{X}}^{n}\right)}{\exp \left\{\rho n R\left(P_{\boldsymbol{x}}, Q_{\hat{X}} \mid \Delta\right)\right\}}\right| \leq 0 .
$$


Combining (10) and (11), we have

$$
G_{\rho}\left(\boldsymbol{x} \mid Q_{\hat{X}}^{n}\right) \doteq \exp \left\{\rho n R\left(P_{\boldsymbol{x}}, Q_{\hat{X}} \mid \Delta\right)\right\}
$$

and thus,

$$
\begin{aligned}
& \sum_{\boldsymbol{x} \in \mathcal{X}^{n}} P_{X^{n}}(\boldsymbol{x}) G_{\rho}\left(\boldsymbol{x} \mid Q_{\hat{X}}^{n}\right) \\
& \doteq \sum_{Q_{X} \in \mathcal{P}_{n}} \exp \left\{n\left[\rho R\left(Q_{X}, Q_{\hat{X}} \mid \Delta\right)-D\left(Q_{X} \| P_{X}\right)\right]\right\} \\
& \doteq \exp \left\{n \max _{Q_{X}}\left[\rho R\left(Q_{X}, Q_{\hat{X}} \mid \Delta\right)-D\left(Q_{X} \| P_{X}\right)\right]\right\} .
\end{aligned}
$$

\section{ACKNOWLEDGMENT}

This work was supported in part by JSPS KAKENHI Grant Number $18 \mathrm{~K} 04141$.

\section{REFERENCES}

[1] J. L. Massey, "Guessing and entropy," in Proc. 1994 IEEE Int. Symp. on Information Theory, p. 204.

[2] E. Arikan, "An inequality on guessing and its application to sequential decoding," IEEE Trans. Inf. Theory, vol. 42, no. 1, pp. 99-105, 1996.

[3] E. Arikan and N. Merhav, "Guessing subject to distortion," IEEE Trans. Inf. Theory, vol. 44, no. 3, pp. 1041-1056, 1998.

[4] S. Salamatian, W. Huleihel, A. Beirami, A. Cohen, and M. Médard, "Why botnets work: Distributed brute-force attacks need no synchronization," IEEE Transactions on Information Forensics and Security, vol. 14, no. 9, pp. 2288-2299, 2019.

[5] A. Rényi, "On measures of entropy and information," in Proc. 4th Berkeley Symp. on Math. Stat. and Prob., 1961, pp. 547-561.

[6] I. Sason and S. Verdú, "Improved bounds on lossless source coding and guessing moments via Rényi measures," IEEE Trans. Inf. Theory, vol. 64, no. 6, pp. 4323-4346, 2018.

[7] S. Kuzuoka, "On the conditional smooth rényi entropy and its applications in guessing and source coding," IEEE Trans. Inf. Theory, vol. 66, no. 3, pp. 1674-1690, 2020.

[8] S. Saito and T. Matsushima, "Non-asymptotic fundamental limits of guessing subject to distortion," in 2019 IEEE International Symposium on Information Theory (ISIT), 2019, pp. 652-656.

[9] M. K. Hanawal and R. Sundaresan, "Guessing revisited: A large deviations approach," IEEE Trans. Inf. Theory, vol. 57, no. 1, pp. 70-78, 2011.

[10] M. M. Christiansen and K. R. Duffy, "Guesswork, large deviations, and Shannon entropy," IEEE Trans. Inf. Theory, vol. 59, no. 2, pp. 796-802, 2013.

[11] M. M. Christiansen, K. R. Duffy, F. du Pin Calmon, and M. Médard, "Multi-user guesswork and brute force security," IEEE Trans. Inf. Theory, vol. 61, no. 12, pp. 6876-6886, 2015.

[12] A. Bracher, A. Lapidoth, and C. Pfister, "Guessing with distributed encoders," Entropy, vol. 21, no. 3, 2019.

[13] N. Merhav and A. Cohen, "Universal randomized guessing with application to asynchronous decentralized brute-force attacks," IEEE Trans. Inf. Theory, vol. 66, no. 1, pp. 114-129, 2020.

[14] J. Ziv and A. Lempel, "Compression of individual sequences via variable-rate coding," IEEE Trans. Inf. Theory, vol. IT-24, no. 5, pp. 530-536, Sep. 1978.

[15] N. Merhav, "Guessing individual sequences: Generating randomized guesses using finite-state machines," IEEE Trans. Inf. Theory, vol. 66, no. 5, pp. 2912-2920, 2020.

[16] N. Merhav and I. Sason, "Some useful integral representations for information-theoretic analyses," Entropy, vol. 22, no. 6, 2020.

[17] I. Csiszár and J. Körner, Information Theory: Coding Theorems for Discrete Memoryless Systems, 2nd ed. Cambridge University Press, 2011.

[18] A. Dembo and I. Kontoyiannis, "Source coding, large deviations, and approximate pattern matching," IEEE Trans. Inf. Theory, vol. 48, no. 6 , pp. 1590-1615, 2002.

[19] E. Yang and Z. Zhang, "On the redundancy of lossy source coding with abstract alphabets," IEEE Trans. Inf. Theory, vol. 45, no. 4, pp. 1092$1110,1999$.
[20] A. W. Marshall, I. Olkin, and B. C. Arnold, Inequalities: Theory of Majorization and Its Applications, 2nd ed. Springer, 2010.

[21] S. Saito, H. Yagi, and T. Matsushima, "Variable-length lossy compression allowing positive overflow and excess distortion probabilities," in 2017 IEEE International Symposium on Information Theory (ISIT), 2017, pp. 1568-1572. 